\title{
Forewarned is forearmed
}

In precise detail, the COVID-19 pandemic currently ravaging the world could not have been predicted. No one could foresee that an RNA virus would leap from a bat into some other intermediate animal carrier, and then into a person, where it would thrive and begin spreading through the human population. The sequence of events depended on chance. Even so, scientists have known that a pandemic of some kind was coming our way, sooner or later.

'Zoonoses' is the term biologists use for diseases caused by viruses or bacteria in other animals. The word comes from Greek - 'zoo' for animals, 'nosos' for diseases. A universe of zoonoses hovers close to humanity as an enduring threat, occasionally signalling its proximity through outbreaks of new infectious diseases. In Europe in 1347, it was the bubonic plague. More recently, the ZIKA virus emerged in 1947, HIV in 1959, and the Ebola virus in 1976. Since then we've seen the SARS and MERS viruses in 2003 and 2013, respectively. After the current coronavirus, there will undoubtedly be others.

In fact, the frequency of pathogens hopping successfully from other species into human beings appears to be increasing - yet another consequence of large-scale human activities on the natural world. Some studies suggest a two- to three-fold increase in the frequency of viral hopping events over the past 40 years, likely due to the large increase in the human population and its expansion into wildlife areas. High-risk zones for new zoonotic infections tend to lie in tropical regions undergoing significant land-use change, and where wildlife biodiversity is high (Allen, T. et al. Nat. Commun. 8, 1124; 2017).

The best way we can protect ourselves from this threat is by building knowledge, and then using it to frame better policies on many fronts. An analogy might be made to NASA's project to map out all the potentially hazardous asteroids, hoping to provide advance warning of impending doom and a chance to avoid it. In the case of emerging infectious diseases, the challenge is to map out hundreds of thousands of unknown pathogens which live in many animal hosts, with the aim of predicting with some accuracy where the next zoonotic outbreak is most likely. It's an onerous and expensive undertaking, yet the cost is small compared to the devastating social and economic consequences of a global pandemic, as we're now learning.
For viruses, a research effort of just this kind has been active for the past decade, although, ironically, the project's funding was terminated just as the COVID-19 virus was emerging. (Apparently, this wasn't due to govenment malfeasance, but simply the ending of the project's five-year schedule; it's now been given an emergency extension for another six months.) In the PREDICT project, researchers sought to identify some of the hundreds of thousands of viral species likely to be living in wild animals. At completion, researchers had identified some 1,100 unique new viruses, provided funding for 60 disease detection laboratories, and trained more than 6,000 people across Asia and Africa, giving them invaluable skills in safe pathogen handling and sample techniques. Another project set to begin in August, called STOP Spillover, aims to carry this work further, intervening at the riskiest human-animal interfaces to improve monitoring efforts and reduce the likelihood of zoonotic spillover.

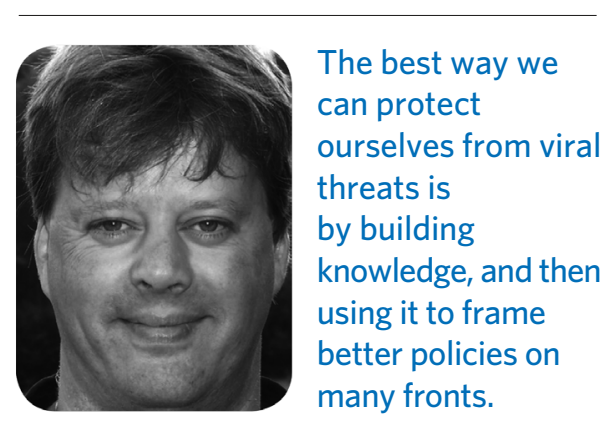

The task of virus identification will also be carried on at a larger scale by the Global Virome Project, which hopes to identify around $99 \%$ of the most important viral diversity from the zoonotic reservoirs in just 10 years. Viruses missed out, the researchers expect, will mostly be those adapted to rare animal species, and so present little opportunity for spillover and public health risk. Out of all the discovered viruses, it's likely that only a small minority will actually have potential to thrive in humans. Even so, data produced by the project could provide a great payoff by enhancing diagnostic capacity in the early stages of a new disease outbreak, thereby aiding the public health response.

Of course, stopping threats from new pathogens, viruses or not, involves more than just finding an accurate list of the pathogens and the animals that harbour them. Many factors influence the likelihood that any pathogen will leap from an animal population into humans. First off is 'pathogen pressure' - the density of pathogen in human proximity at a particular point in space and time. This is determined by the distribution of people as well as infected animals, the density of pathogen within their hosts, and also how routinely pathogen escapes from the host - for example, during human handling or food production. Other factors include how long the pathogen can survive outside of its host, the degree of human exposure (including frequency and duration) and the prevailing biological barriers to successful infection presented by the potential human host.

Mapping out high-risk zones for such spillover events requires detailed analysis of all these factors, which shift in space and time, making eventual monitoring to reduce spillover events very challenging (Plowright, R. et al. Nat. Rev. Microbiol. 15, 502-510; 2017). The immense difficulty of getting a decent picture of all these factors as they continually change is a big reason why we're still not able to predict when, where or from what species the next emerging virus will break out. It's why our human approach to controlling epidemics has largely been passive and reactive - wait for a new infectious agent to appear, and then try to stop it. Of course, public health officials have also tried to be prepared and to build resilience, knowing full well that that more epidemics are coming, even if we don't know from where or when.

But there's no reason why humanity can't adopt a more pro-active strategy. Doing so requires knowledge and determination. With advancing biotechnologies over the past two decades, we've created the possibility of mapping out most human pathogens. We've also vastly expanded our computational capacity to understand the distributions and movements of human and animal populations in quantitative detail, and so can at least imagine being able to predict the most likely zones for spillover events in something like real time. We're not there yet, but in 20 years could be.

\section{Mark Buchanan}

Published online: 7 May 2020 https://doi.org/10.1038/s41567-020-0900-2 